\title{
LTE RF receiver modeling and each part testing with MATLAB simulink
}

\author{
Lec. Fatime faydhe al-azzawi
}

Middle Technical University (MTU), Institute of Technology, Iraq

\begin{tabular}{|c|c|}
\hline Article Info & ABSTRACT \\
\hline Article history: & Fourth generation cellular network based on Long Term Evaluation (LTE) \\
\hline Received Sep 16, 2018 & $\begin{array}{l}\text { air interface. LTE is specifically designed for packet data communications, } \\
\text { where the emphasis of the technologies high spectral efficiency, high peak }\end{array}$ \\
\hline Revised Nov 17, 2018 & data rates, low latency, and frequency flexibility. In this paper a LTE \\
\hline Accepted Feb 22, 2019 & $\begin{array}{l}\text { physical layer design and implemented using MATLAB codes and Simulink, } \\
\text { where MATLAB codes generate the } 64 \text { QAM transmitting signal and }\end{array}$ \\
\hline Keywords: & $\begin{array}{l}\text { simulating LTE frames while Simulink model perform the physical layer } \\
\text { which including the band limiting, free space path loss adding, channel, RF }\end{array}$ \\
\hline $\begin{array}{l}\text { LTE } \\
\text { Physical layer } \\
\text { RF receiver }\end{array}$ & $\begin{array}{l}\text { receiver, demodulation, 10-bit ADC, DC offset cancellation, saving data to } \\
\text { preform EVM measurements, and viewing the spectrum signal after each } \\
\text { block, finally QAM with multi-order tested under AWGN. }\end{array}$ \\
\hline
\end{tabular}

\section{Corresponding Author:}

Lec. Fatime faydhe al-azzawi,

Middle Technical University (MTU),

Institute of Technology, Baghdad, Iraq.

Email: Fatima.faydhe@mtu.edu.iq

\section{INTRODUCTION}

Long-term Evaluation (LTE) is the air interface supporting fourth generation cellular networks. LTE is specifically designed for packet data communications, where the emphasis of the technology is high spectral efficiency, high peak data rates, low latency, and frequency flexibility. The LTE specifications were developed by the Third Generation Partnership Project e(3GPP) [1], [2].

The LTE standards continue to evolve over multiple releases to capture requirements that lead to improved data throughput, lower latencies, and increasingly flexible configurations. After the release is frozen, 3GPP continues revisions of the associated standards to correct errors and fill in omissions, but no new features are introduced [3]. The Releasei8 functionality set was frozen in the March 2009 standards release (SA\#43). The release consisted of a completely new radio interface and core network, which enabled substantially improved data performance compared with previous systems. The Release 9 functionality set was frozen in the March 2010 standards release (SA\#47). LTE Releasei9ibrought refinements to LTE Release 8, and it introduced some new service features and network architecture improvements. The Releasei10 functionality set was frozen in the June 2011istandards release (SA\#52). LTE Release 10 is considered to be the start of LTE -Advanced. It significantly improved data throughput and extended cell coverage.

The Release 11 functionality set was frozen in the March 2013 standards release (SA\#59). LTE Releasei11 included refinements to existing Release 10icapabilities. The Releasei12 functionality set was frozen in the Marchi2015 standards release (SA\#67), where Enhanced small cells for LTE, introducing a number of features to improve the support of Het Nets. The LTE standard releases from 13 onwards are known as LTE-Advanced Pro. New working group introduced for programming of mission critical applications. LTE operation in a combination of licensed and unlicensed spectrum. Inter site carrier aggregation, to coordinate the capabilities and backhaul of adjacent cells. Enhanced machine-to- 
machine (M2M) communication, also referred to as machine- type communication (MTC). Interworking with Wi-Fi, licensed assisted access (at $5 \mathrm{GHz}$ ). Indoor positioning. Further enhancements of public safety features, such as D2D and ProSe, which include small-cell dual-connectivity and architecture changes. Single-cell point to multipoint. New antenna techniques, such as 3D/FD- MIMO, which include study of high-order MIMO systems with up to 64 antenna ports. Advanced receivers to maximize the potential of large cells. Work on latency reduction. [4,5]. In this paper LTE RF receiver will be design and implemented with MATLAB code and Simulink and illustrate the spectrum of signals after each state in the receiver finally QAM modulation examined under AWGN with multi order.

\section{RADIO ACCESS NETWORK OF LTE}

The LTE radio access network is comprised of the following protocol entities. Packet Data Convergence Protocol (PDCP), Radio Link Control (RLC), Medium Access Control (MAC) and the Physical Layer (PHY) [6], [7].

The first three protocol entities handle tasks such as header compression, ciphering, segmentation and concatenation, and multiplexing and de-multiplexing. The physical layer handles coding and decoding, modulation and demodulation, and antenna mapping. Figure 1 shows the delineation between the physical layer and higher layers.

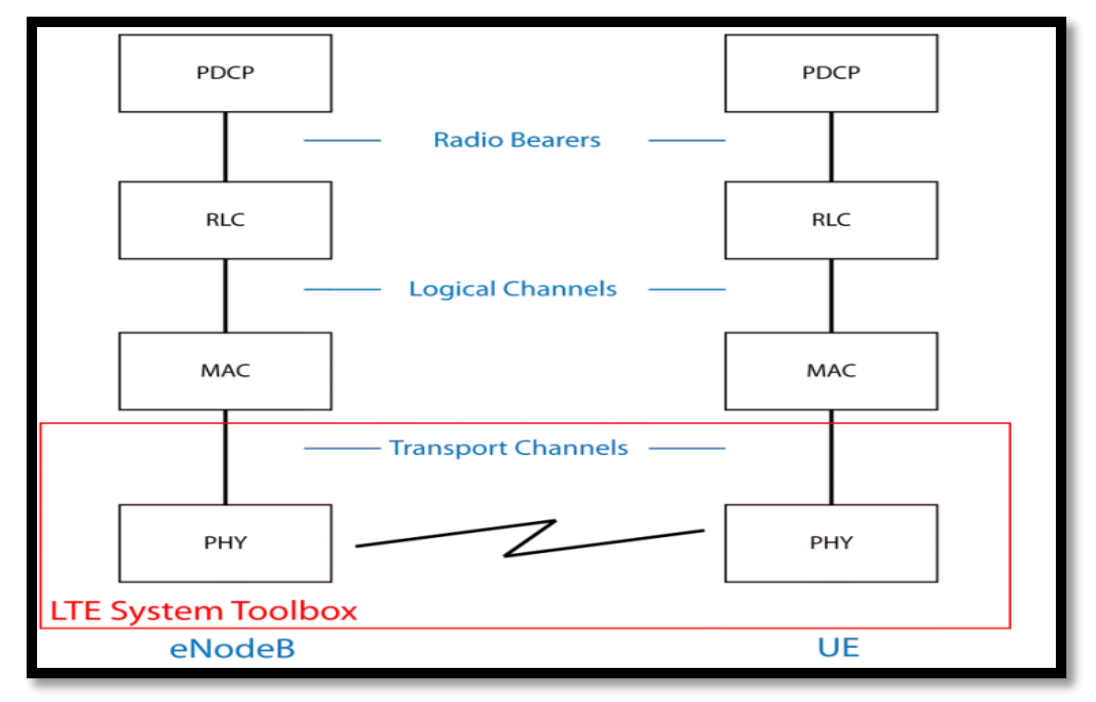

Figure 1. Radio access network of LTE

From Figure 1 LTE System focuses on the physical layer, which is highlighted in red in the preceding figure. It also supports interfacing with portions of the RLC and MAC layers, which are highlighted in blue. The primary features of the LTE physical layer are OFDM modulation, including the time - frequency structure of the resource blocks, adaptive modulation and coding, hybrid- ARQ, and MIMO.

\section{MODEL DESCRIPTION}

The figure below shows the main parts of the proposed model. An LTE waveform is generated using the LTE System Toolbox. This waveform is filtered and transmitted through a propagation channel before feeding it to the RF receiver model implemented with RF Block set. This model is based on commercially available parts. EVM figures are then provided for the output of the RF receiver [6], [8].

The error vector magnitude or EVM (sometimes also called relative constellation error or RCE) is a measure used to quantify the performance of a radio transmitter or receiver. A signal sent by an ideal transmitter or received by a receiver would have all points precisely at the ideal locations, however various imperfections in the implementation (such as carrier leakage, low image rejection ratio, phase noise etc.) cause the actual constellation points to deviate from the ideal locations. Informally, EVM is a measure of how far the points are from the ideal locations [6], [8], [9]. 


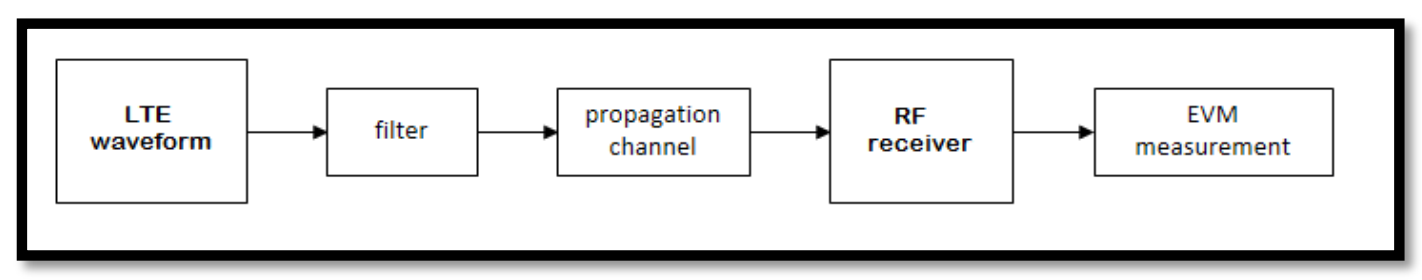

Figure 2. LTE RF receiver

Simulink model shown in Figure 3 is the LTE RF receiver where this model includes the following components: Reading the LTE waveform and the sampling period from the workspace, Band limiting filtering, Channel model: this includes free space path loss and AWGN, RF receiver, including direct conversion demodulator, $\mathrm{ADC}$ and $\mathrm{DC}$ offset cancellation.

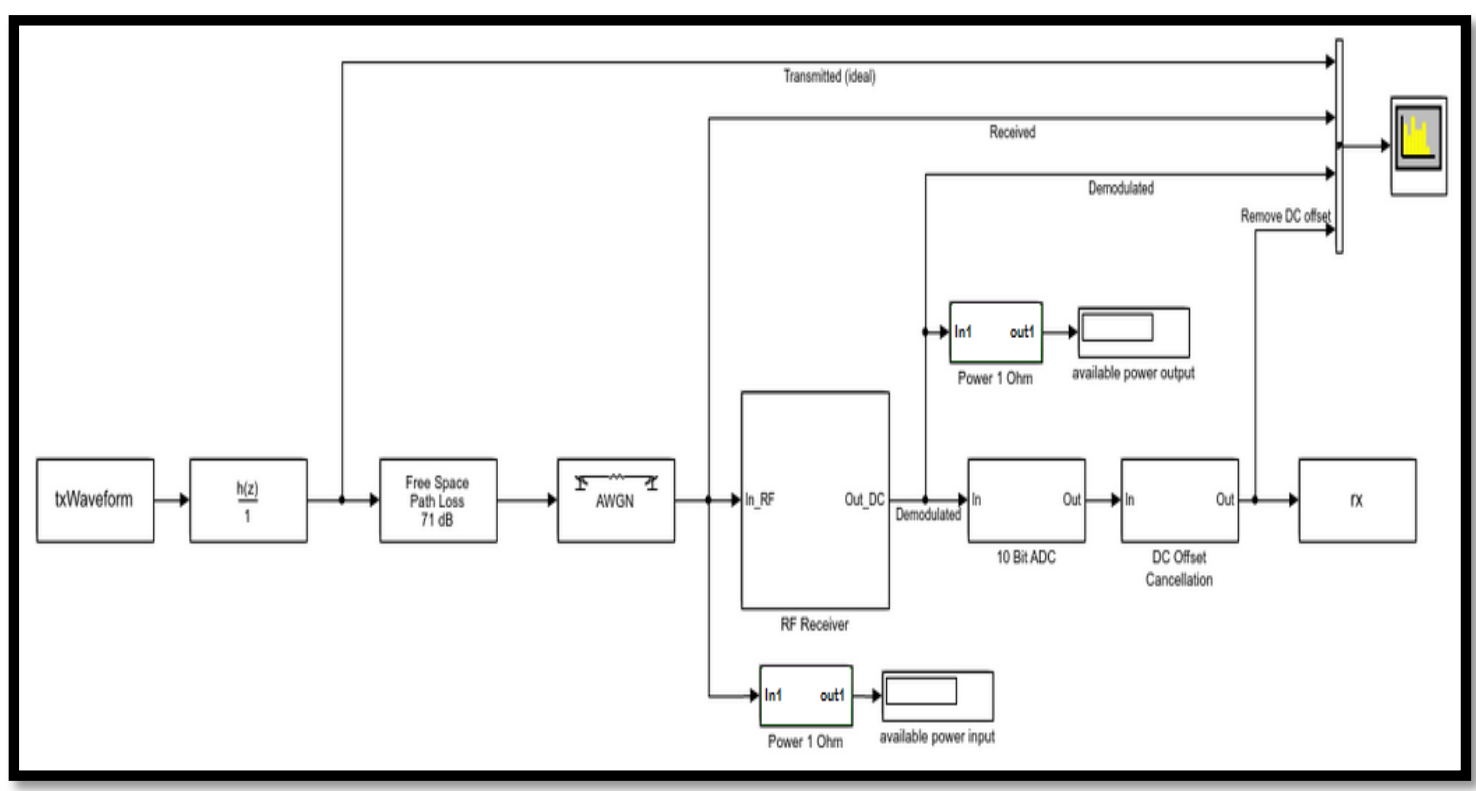

Figure 3. LTE RF receiver simulink modal

First block in the Simulink model is for generating the LTE waveform using the LTE System Toolbox. using the reference measurement channel (RMC) R.6 as defined in TSi36.101 [4], [5]. This RMC specifies a 25 resource elements (REs) bandwidth, equivalent to $5 \mathrm{MHz}$. A 64 QAM modulation is used because higher- order QAM can deliver more data less reliably than lower-order QAM, for constant mean constellation energy. Using higher-order QAM without increasing the bit error rate requires a higher signalto - noise ratio (SNR) by increasing signal energy, reducing noise, or both [6], [10]. If data- rates beyond those offered by 8-PSK are required, it is more usual to move to QAM since it achieves a greater distance between adjacent points in the I-Q plane by distributing the points more evenly. The complicating factor is that the points are no longer all the same amplitude and so the demodulator must now correctly detect both phase and amplitude, rather than just phase [7], [11], [12]. All REs are allocated. Additionally, Orthogonal Channel Noise Generation (OCNG) noise is enabled in unused REs.

Discreet FIR filter is used for band limiting where Independently filter each channel of the input over time, free space path loss block used to reduce the amplitude of the input signal by the amount specified. The reciprocal of the loss is applied as a gain, e.g., a loss of $+20 \mathrm{~dB}$, which reduces the signal by a factor of 10 , corresponds to a gain value of 0.1 [13]. AWGN is add to the signal as noise channel then the RF receiver which shown in Figure 4. 


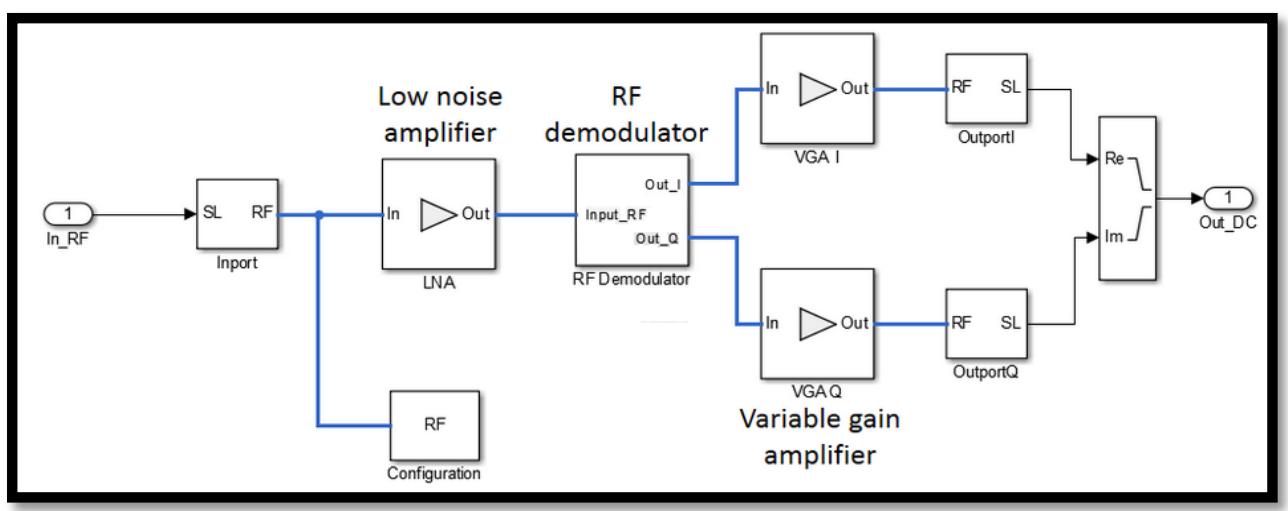

Figure 4. RF receiver simulink model

The RF demodulator includes the following components and is shown in Figure 5. Local oscillator (LO) and phase noise model Phase shift for I and Q components generator Mixers

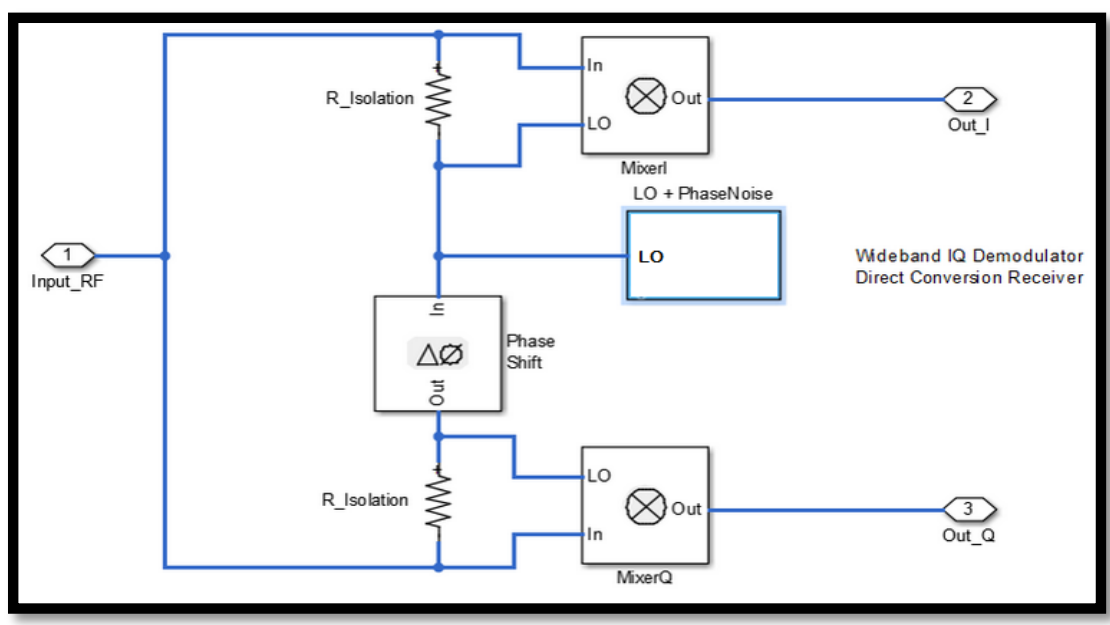

Figure 5. RF demodulator simulink block

The 10-bit ADC introduce quantization and saturation on complex signals with Full Scale Power 30dBm, Number of Quantization Levels 10, the DC offset cancellation is to remove the DC offset by averaging the input signal. The output of the Simulink model is stored in the variable $|\mathrm{rx}|$, which is available in the workspace. Any delays introduced to this signal are removed after performing synchronization. The EVM is measured on the resulting waveform.

Simulates the specified number of frames is done in two stages: Simulate the first frame Simulate the rest of the frames in a loop, the reason for splitting the processing in these two stages is to simplify the code. During the processing of the first frame we need to take into account the delay of the band limiting filter. This is not the case for subsequent frames, since the filter state is maintained between frames. Therefore, the length of the first frame has to be increased slightly to take into account the delay introduced by the filter.

\section{RESULTS}

Using spectrum analyzer after each block in the Simulink model of Figure 3 to view the spectrum changing of the signal due to the function of each block. From Figure 7 as compared to Figure 6, the spectrum after band limitation illustrate increasing in $\mathrm{dBm}$ between main and side lobes where the signal reaches to ideal spectrum. 


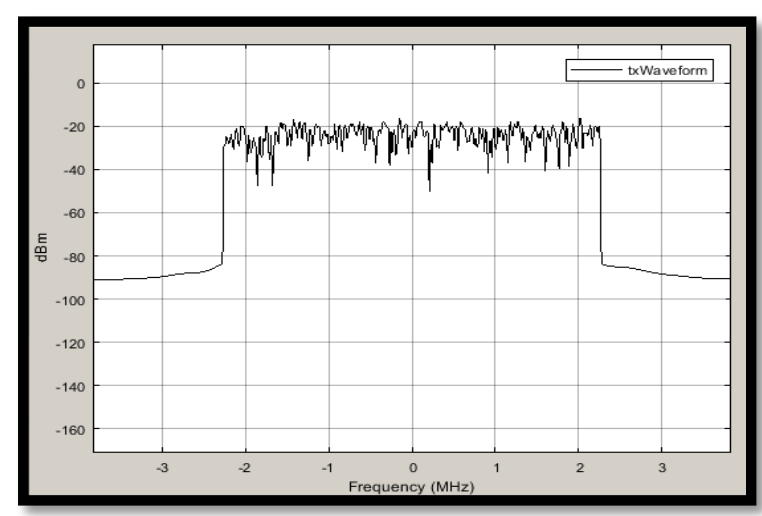

Figure 6. TxWaveform spectrum

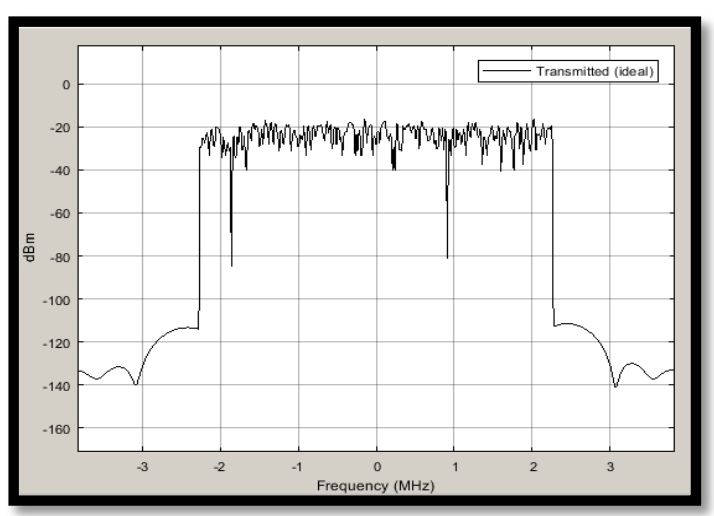

Figure 7. Band limited signal spectrum

After free space path loss, the spectrum reduces $71 \mathrm{dBm}$ as shown in Figure 8. The received signal which is the signal after AWGN channel is illustrated in Figure 9, where the spectrum viewing the existence of AWGN on the spectrum as compared to spectrum in Figure 8. Figures 10-12 illustrate the demodulated spectrum, 10-bit ADC and removing DC offset cancellation respectively.

As mention above Simulates the specified number of frames is done in two stages: Simulate the first frame Simulate the rest of the frames in a loop, The EVM is measured on the resulting waveform. As in Figure 13 where EVM peak is measured with respect to number of frames while Figure 14 shows the EVM RMS with respect to frames number.

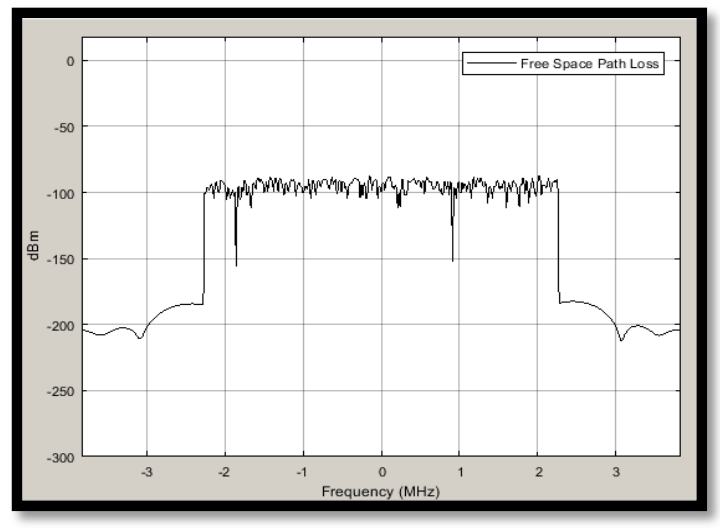

Figure 8. Free space path loss signal spectrum

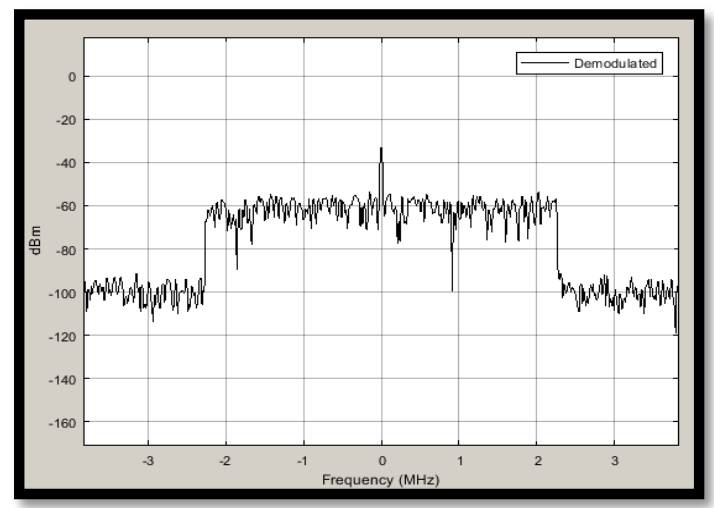

Figure 10. Demodulated spectrum signal

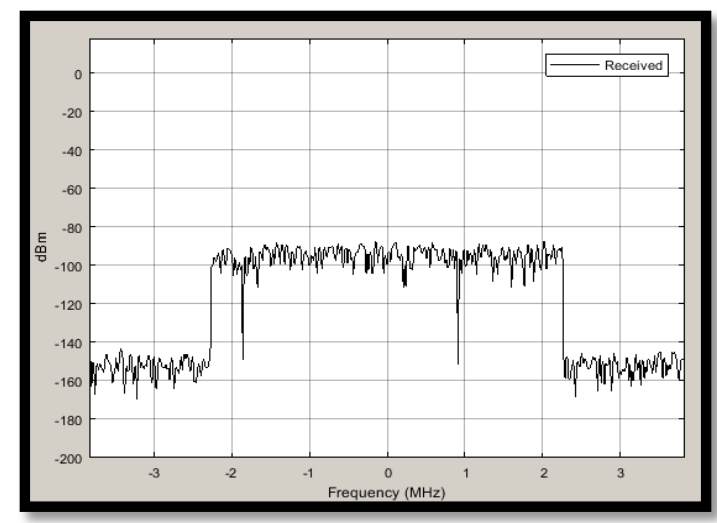

Figure 9. Received spectrum signal

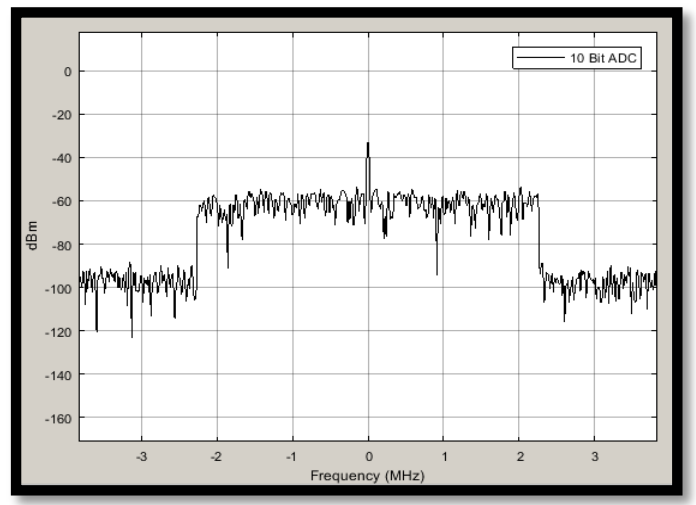

Figure 11. After ADC spectrum signal 


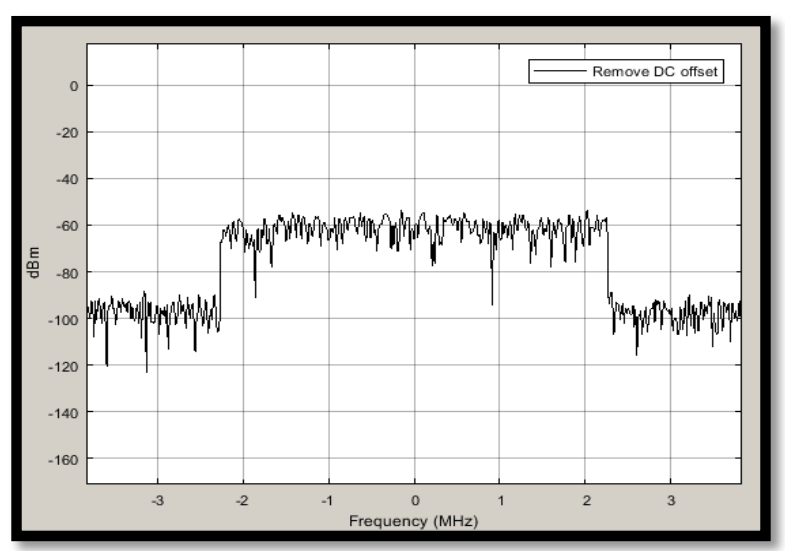

Figure 12. After removing DC offset spectrum signal

QAM modulation technique that used to generate the transmitting signal and in the demodulation process have been examined in AWGN channel with multi order, Figure 15 shows QAM modulation with various order under AWGN channel. From the figure 64 QAM is the most suitable order for propose model for reasons mansion above.



Figure 13. The measured peak and EVM for each simulated frame

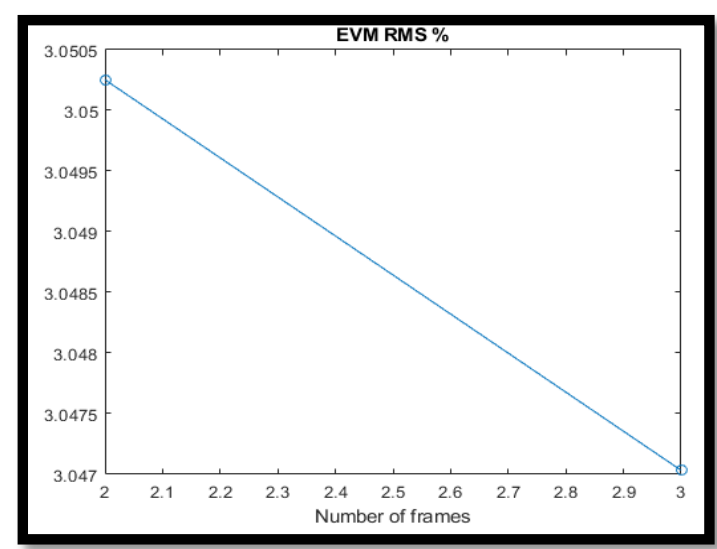

Figure 14. The measured peak and EVM RMS for each simulated frame

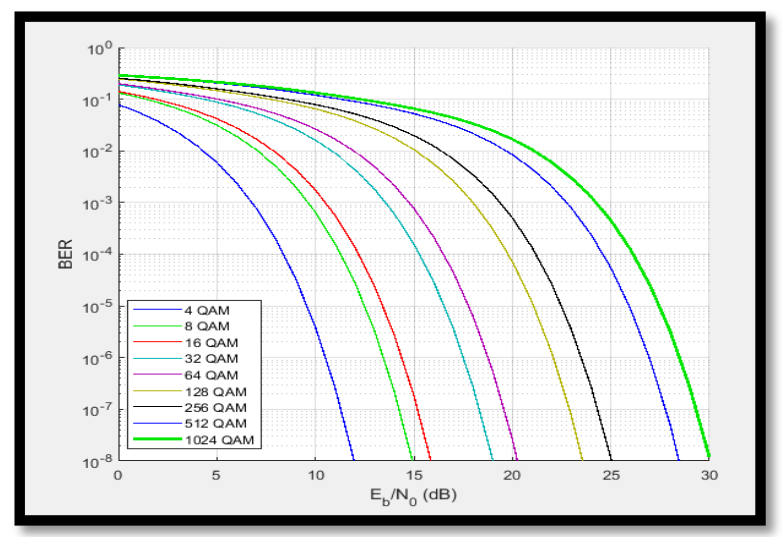

Figure 15. QAM modulation with multi order under AWGN channel 


\section{CONCLUSION}

In this paper LTE RF receiver Simulink model have been design and implanted in MATLAB Simulink and codes to perform the physical layer of 4G LTE air interface where 64 QAM modulation technique used to increasing the bit error rate requires a higher signal - to-noise ratio (SNR) by increasing signal energy, reducing noise, or both. FIR filter used for band limiting to reach the idealism of transmitted spectrum signal, free space path loss reduces the spectrum by $71 \mathrm{dBm}$, the received signal demodulated, converted analog by 10 bit $-\mathrm{ADC}$, the DC offset removed and finally the data saved to perform the EVM measurements according to number of frames which simulated using MATLAB codes, the LTE standard introduced for programming of mission critical applications. LTE operation in a combination of licensed and unlicensed spectrum.

\section{REFERENCES}

[1] Gautam Siwach, Dr. Amir Esmailpour "LTE Security Potential Vulnerability and Algorithm Enhancements", IEEE Canadian Conference on Electrical and Computer Engineering," IEEE CCECE, Toronto, Canada, May 2014.

[2] Sajal K. Das, "Mobile Terminal Receiver Design: LTE and LTE-Advanced", ISBN 978-1-1191-0730-9. John Wiley \& Sons, April 2016.

[3] Guowang Miao, Jens Zander, Ki Won Sung, and Ben Slimane, "Fundamentals of Mobile Data Networks", Cambridge University Press, ISBN 1107143217, 2016.

[4] Nohrborg, Magdalena, for 3GPP. "LTE Overview." 3GPP, A Global Initiative, THE Mobile Broadband Standard, August 2013.

[5] Dahlman, E., Parkvall, S., and Sköld, J. 4G LTE/LTE-Advanced for Mobile Broadband. Kidlington, Oxford: Academic Press, 2011. pp. 112-118.

[6] McKinley et al "EVM Calculation for Broadband Modulated Signals", 64th ARFTG Conf. Dig., Orlando, FL, pp. 45-52, Dec. 2004.

[7] Cho, Kyongkuk; Yoon, Dongweon "On the General BER Expression of One- and Two-Dimensional Amplitude Modulations". IEEE Trans. Commun, July 2002.

[8] Jonqyin (Russell) Sun "Linear diversity analysis for QAM in Rician fading channels", IEEE WOCC 2014

[9] Walko, John "NXP powers ahead with programmable LTE modem", EETimes, January 30, 2008.

[10] Lynnette Luna, "Alcatel-Lucent says new antenna technology boosts LTE, $3 G$ data speeds". Fierce Broadband Wireless. Retrieved March 24, 2010.

[11] Dan Jones (16 October 2012). "Defining 4G: What the Heck Is LTE TDD?". Light Reading. Retrieved 9 January 2014.

[12] Ian Poole. "LTE-FDD, TDD, TD-LTE Duplex Schemes". Radio-electronics.com. Retrieved 9 January 2014.

[13] Mallinson, Keith. "On Cumulative mobile-SEP royalties" (PDF). Wise Harbor. Retrieved January 23, 2017. 